\title{
Angiotensin II receptor blocker is a renoprotective remedy for metabolic syndrome
}

\author{
Tadashi Sofue and Hideyasu Kiyomoto
}

Hypertension Research (2009) 32, 735-737; doi:10.1038/hr.2009.123; published online 7 August 2009

$\mathrm{O}$ besity leads to a high risk of developing various diseases, such as type II diabetes, hypertension, dyslipidemia and ischemic heart disease. Metabolic syndrome is characterized by visceral obesity, glucose intolerance, dyslipidemia and hypertension. Insulin resistance and activation of the sympathetic nerve system were often observed in individuals with metabolic syndrome. Although the absence of leptin or its receptor causes severe obesity and hyperphagia in humans and in rodents, ${ }^{1}$ leptin itself has not yet been used as a practical medicine for metabolic syndrome. As the prevalence of metabolic syndrome has been increasing along with the development of modern conveniences throughout the world, establishment of an effective prevention against metabolic syndrome is greatly anticipated.

There are few clinical studies on metabolic syndromes treated with angiotensin II receptor blocker (ARB). On the other hand, largescale clinical trials for diabetes mellitus have shown that aggressive treatments with ARB were effective in reducing microalbuminuria, or that they suppressed the progression to overt diabetic nephropathy. ${ }^{2-5}$ Additional studies for advanced renal insufficiency have also shown that ARBs inhibited the transition from overt nephropathy to end-stage renal disease in type II diabetic patients. ${ }^{6-8}$ These data suggested that treatments with ARB ameliorated each stage of diabetic nephropathy other than antihypertensive properties (Figure 1). Therefore, ARB for diabetic nephropathy has established an indispensable therapy in large-scale clinical studies ${ }^{2-8}$

T Sofue and $\mathrm{H}$ Kiyomoto are at the Division of Nephrology and Dialysis, Department of CardioRenal and CerebroVascular Medicine, Faculty of Medicine, Kagawa University, Kagawa, Japan

E-mail: kiyo@med.kagawa-u.ac.jp and stage-matched experimental studies. ${ }^{9-12}$ Moreover, the Randomised Olmesartan and Diabetes Microalbuminuria Prevention (ROADMAP) study is ongoing, and its purpose is to determine whether ARB would be able to prevent the occurrence of microalbuminuria in type II diabetic patients with normoalbuminuria. ${ }^{13}$

The renin-angiotensin-aldosterone system (RAAS) in the kidney is believed to be involved in a crucial network that has influence in the development of renal injury. It is reported that increases in intrarenal angiotensin II induce renal injury in several animal models. ${ }^{9,10,14-17}$ Renal injury in diabetic nephropathy was formerly believed to be less involved with RAAS due to the fact that plasma renin activity in patients with diabetes mellitus is relatively lower than in normal individuals. However, Nagai et al. ${ }^{9}$ proved that intrarenal angiotensin II contents in prediabetic kidney were already higher than those in nondiabetic rats in spite of the plasma angiotensin II level being low. In addition, Nishiyama et al. ${ }^{10}$ reported that ARB treatment restored angiotensin II contents in the kidney concomitant with the prevention of microalbuminuria.

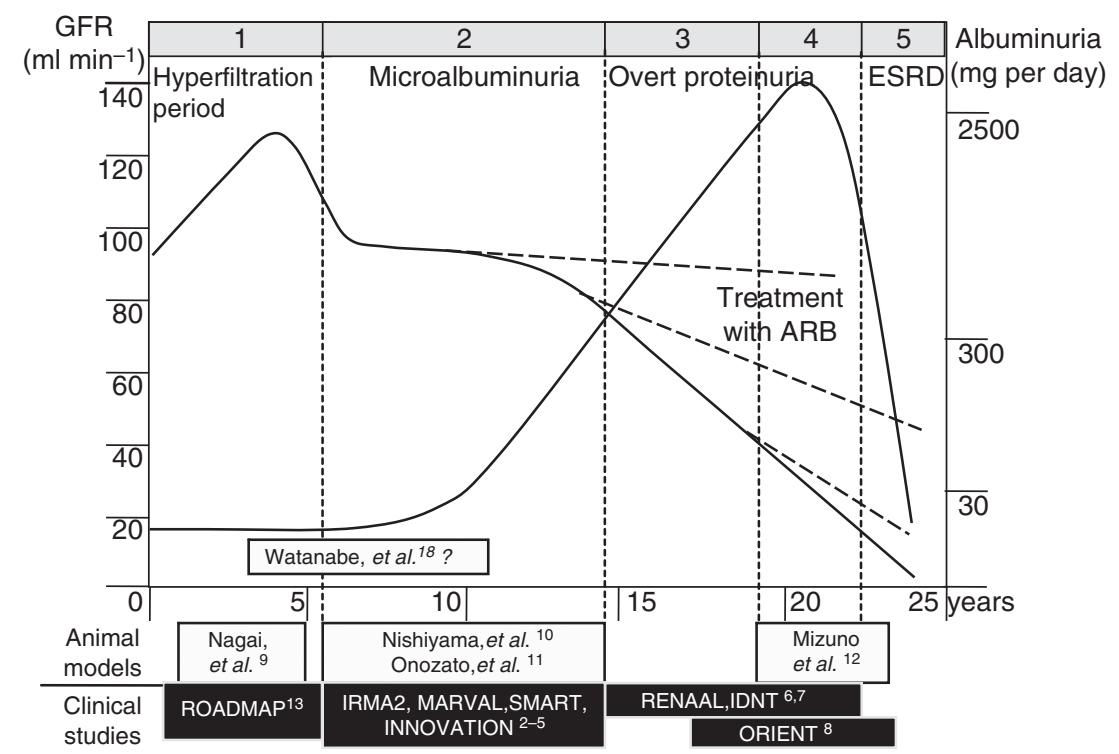

Figure 1 The stages of diabetic nephropathy in humans and the relationships between therapeutical interventions by ARBs and renal functions. Renal functions are determined by GFR (left axis) and/or by the extent of albuminuria (right axis). Several large clinical studies and experimental studies matched with the natural clinical stage of diabetic nephropathy have been carried out for a decade. ${ }^{2-13}$ Broken lines indicate the supposed course after starting interventional treatments of ARBs. An experimental model of metabolic syndrome is similar but not identical to diabetic nephropathy. ${ }^{18}$ Numbers in parentheses indicate the reference number. GFR, glomerular filtration rate; ARB, angiotensin II receptor blocker; ESRD, end-stage renal disease. 
In this issue of Hypertension Research, Watanabe et al. ${ }^{18}$ report that treatment with olmesartan effectively decreases urinary protein level and ameliorates pathological damages in the kidney of spontaneously hypertensive (SHR)/NDmcr-cp rats, an experimental model for metabolic syndrome. SHR/NDmcr-cp rats have a genetic mutation of leptin receptor genes, so that they develop severe obesity and hyperphagia similar to $o b / o b$ mice. Instead of type 2 diabetic rats (9-12), the SHR/NDmcr-cp rats showed more prominent accumulation of visceral fat than those type 2 animals, and were considered as the better model for advanced metabolic syndrome. In their study, renal injury in SHR/NDmcr-cp rats represented a histological change similar to early features of diabetic nephropathy, such as mesangial matrix expansion and thickening of glomerular basement membrane, in an analysis using electron microscopy. However, they excreted urinary protein before renal pathological changes at 9 weeks of age when they seemed to be in an early stage of metabolic syndrome. Not only olmesartan but also hydralazine was able to decrease urinary protein excretion; therefore, antihypertension treatment was essential for metabolic syndrome at all cost. Nevertheless, the decrease in urinary protein excretion was more remarkable in olmesartan-treated animals at the end of the study. Although plasma angiotensin II levels were similar to each other in all experimental groups, administration of only olmesartan ameliorated the pathological abnormalities of renal glomerular and tubulointerstitial injuries with the suppression of TGF- $\beta$ (tumor growth factor- $\beta$ ) and type IV collagen in rats with metabolic syndrome. Interestingly, no remarkable change was observed in electron microscopic analysis in SHR/NDmcr-cp rats at 9 weeks of age, despite these animals showing overt proteinuria. In general, the incidence of proteinuria and/or microalbuminuria is usually attributed to the lesions of the glomerular basement membrane involved in podocyte ultrastructural abnormality. For instance, a recent report showed that a significant accumulation of cytoplasmic granules with albumin immunosignals in podocyte was observed in the early phase of type II diabetic rats and that strict angiotensin II blockade by telmisartan prevented the incidence of microalbuminuria and podocyte abnormalities. ${ }^{10}$

Those discrepancies between diabetic nephropathy ${ }^{9-12}$ and metabolic syndrome ${ }^{18}$ in animal models might derive from the influence of glomerular hyperfiltration, which is caused by obesity. Hyperfiltration

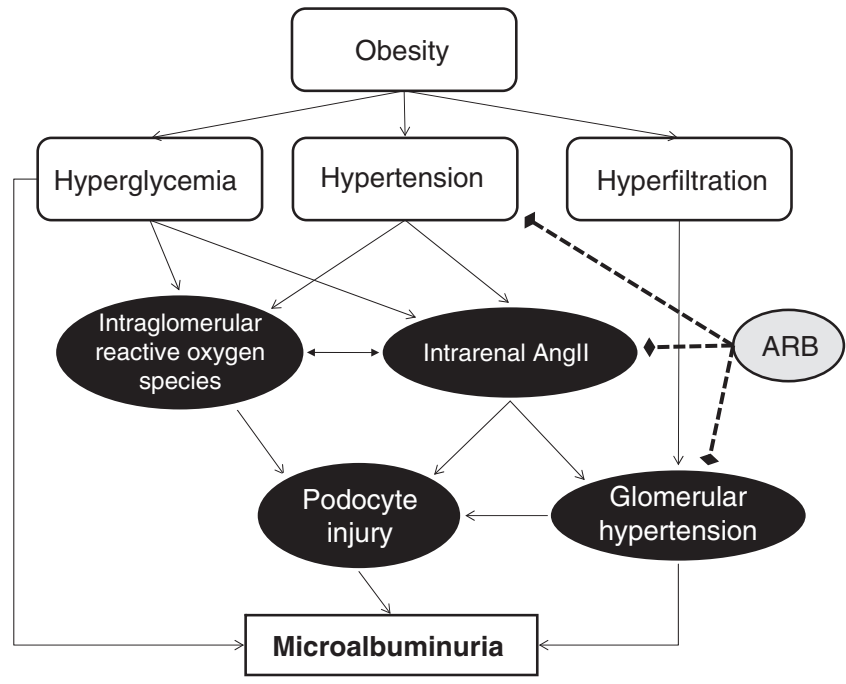

Figure 2 The hypothetical mechanisms for incidence of microalbuminuria in metabolic syndrome. Hyperglycemia and hypertension cause the accumulation of intrarenal angiotensin II contents and intraglomerular reactive oxygen species. Intrarenal angiotensin II contents and intraglomerular reactive oxygen species directly lead to podocyte dysfunction, which links to microalbuminuria. Three broken lines indicate the inhibitory effects of ARB in metabolic syndrome. Ang II, angiotensin II; ARB, angiotensin II receptor blocker.

with obesity independently increases glomerular arterial pressure and leads to microalbuminuria without podocyte injury. ${ }^{19}$ We then summarized the relationships between clinical $^{2-8,13}$ or experimental ${ }^{9-12}$ interventions by ARBs and the natural course of diabetic nephropathy in Figure 1. Diabetes nephropathy in humans is usually determined by the glomerular filtration rate and/or the extent of albuminuria (proteinuria). As many reports suggested that interventional treatments of ARBs improved the renal function in any phase of diabetic nephropathy, the renoprotective effects of ARBs would be expected as soon as possible to initiate medicines. In metabolic syndrome, ARB also prevented the progression of albuminuria and pathological abnormalities; ${ }^{18}$ however, we could not accurately determine a suitable period for diabetic nephropathy for this SHR/NDmcr-cp rat model, because renal injury and albuminuria occurred much earlier than we expected in diabetic nephropathy (Figure 1). We therefore consider this experimental model for metabolic syndrome to be similar but not identical to diabetic nephropathy, rather it might be close to obesity-induced secondary nephrosclerosis.

Several candidates may contribute toward causing microalbuminuria in metabolic syndrome (Figure 2). Hypertension accompanied with a disorder of RAAS and hyperglycemia not only impairs insulin signaling but also induces oxidative stress in the glomeruli. Generated reactive oxygen species may additionally accelerate podocyte damage through a loss of slit pore diaphragm integrity. ${ }^{16}$ Patients who have metabolic syndrome often show glomerular hyperfiltration as a risk for cardiovascular event. ${ }^{20}$ The hemodynamic action of angiotensin II has an important role in glomerular damages. Podocyte injury, which is directly linked to microalbuminuria, may be affected by an increase in glomerular capillary pressure through the constriction of efferent arterioles. Moreover, the accumulation of intrarenal angiotensin II causes an increase in angiotensin II type 1 receptor (AT1R) gene expression similar to an autocrine loop of angiotensin II. ${ }^{15,17}$ We now recognize that $\mathrm{ARBs}$ such as olmesartan have so-called 'beyond antihypertensive effects' by blocking AT1R-mediated action in metabolic syndrome. ${ }^{18}$ Taken together, we finally conclude that ARB is a putative panacea for modern life at present.

As shown in Figure 1, large-scale clinical studies using ARBs have been conducted in diabetic nephropathy, ${ }^{2-8,13}$ whereas there are still few evidences in metabolic syndrome treated with ARB. Further investigations are necessary to clarify the mechanisms relating to how metabolic syndrome develops renal injury.

\footnotetext{
1 Farooqi IS, Jebb SA, Langmack G, Lawrence E, Cheetham $\mathrm{CH}$, Prentice AM, Hughes IA, McCamish MA, O'Rahilly S. Effects of recombinant leptin therapy in a child with congenital leptin deficiency. $N$ Engl J Med 1999; 341: 879-884.
} 
2 Lewis EJ, Hunsicker LG, Clarke WR, Berl T, Pohl MA Lewis JB, Ritz E, Atkins RC, Rohde R, Raz I. Renoprotective effect of the angiotensin-receptor antagonist irbesartan in patients with nephropathy due to type 2 diabetes. N Engl J Med 2001; 345: 851-860.

3 Viberti G, Wheeldon NM. Microalbuminuria reduction with valsartan in patients with type 2 diabetes mellitus: a blood pressure-independent effect. Circulation 2002; 106: 672-678.

4 Uzu T, Sawaguchi M, Maegawa H, Kashiwagi A. Impact of renin-angiotensin system inhibition on microalbuminuria in type 2 diabetes: a post hoc analysis of the Shiga Microalbuminuria Reduction Trial (SMART). Hypertens Res 2008; 31: 1171-1176.

5 Makino $\mathrm{H}$, Haneda M, Babazono T, Moriya T, Ito S, Iwamoto $\mathrm{Y}$, Kawamori R, Takeuchi M, Katayama S. The telmisartan renoprotective study from incipient nephropathy to overt nephropathy - rationale, study design, treatment plan and baseline characteristics of the incipient to overt: angiotensin II receptor blocker, telmisartan, Investigation on Type 2 Diabetic Nephropathy (INNOVATION) Study. J Int Med Res 2005; 33: 677-686.

6 Brenner BM, Cooper ME, de Zeeuw D, Keane WF, Mitch WE, Parving HH, Remuzzi G, Snapinn SM, Zhang Z, Shahinfar S. Effects of losartan on renal and cardiovascular outcomes in patients with type 2 diabetes and nephropathy. N Engl J Med 2001; 345: 861-886.

7 Parving $\mathrm{HH}$, Lehnert $\mathrm{H}$, Brochner-Mortensen J, Gomis $\mathrm{R}$, Andersen S, Arner P. The effect of irbesartan on the development of diabetic nephropathy in patients with type 2 diabetes. N Engl J Med 2001; 345: 870-878.
8 Imai $\mathrm{E}$, Ito $\mathrm{S}$, Haneda $\mathrm{M}$, Chan JC, Makino $\mathrm{H}$. Olmesartan reducing incidence of endstage renal disease in diabetic nephropathy trial (ORIENT): rationale and study design. Hypertens Res 2006; 29 . 703-709.

9 Nagai Y, Yao L, Kobori H, Miyata K, Ozawa Y, Miyatake A, Yukimura T, Shokoji T, Kimura S, Kiyomoto H, Kohno $M$, Abe $Y$, Nishiyama A. Temporary angiotensin II blockade at the prediabetic stage attenuates the development of renal injury in type 2 diabetic rats. J Am Soc Nephrol 2005; 16: 703-711.

10 Nishiyama A, Nakagawa T, Kobori H, Nagai Y, Okada $\mathrm{N}$, Konishi Y, Morikawa T, Okumura M, Meda I, Kiyomoto $\mathrm{H}$, Hosomi N, Mori T, Ito S, Imanishi M. Strict angiotensin blockade prevents the augmentation of intrarenal angiotensin II and podocyte abnormalities in type 2 diabetic rats with microalbuminuria. J Hypertens 2008; 26: 1849-1859.

11 Onozato ML, Tojo A, Goto A, Fujita T, Wilcox CS. Oxidative stress and nitric oxide synthase in rat diabetic nephropathy: effects of ACEI and ARB. Kidney Int 2002; 61: 186-194.

12 Mizuno M, Sada T, Kato M, Fukushima Y, Terashima H, Koike $\mathrm{H}$. The effect of angiotensin II receptor blockade on an end-stage renal failure model of type 2 diabetes. J Cardiovasc Pharmacol 2006; 48: 135-142.

13 Haller H, Viberti GC, Mimran A, Remuzzi G, Rabelink AJ, Ritz E, Rump LC, Ruilope LM, Katayama S, Ito S, Izzo Jr JL, Januszewicz A. Preventing microalbuminuria in patients with diabetes: rationale and design of the Randomised Olmesartan and Diabetes Microalbuminuria Prevention (ROADMAP) study. J Hypertens 2006; 24: 403-408.
14 Navar LG, Harrison-Bernard LM, Nishiyama A, Kobori $\mathrm{H}$. Regulation of intrarenal angiotensin II in hypertension. Hypertension 2006; 39: 316-322.

15 Suzuki K, Han GD, Miyauchi N, Hashimoto T, Nakatsue T, Fujioka Y, Koike H, Shimizu F, Kawachi $\mathrm{H}$. Angiotensin II type 1 and type 2 receptors play opposite roles in regulating the barrier function of kidney glomerular capillary wall. Am J Pathol 2007; 170: 1841-1853.

16 Whaley-Connell A, Habibi J, Wei Y, Gutweiler A, Jellison J, Wiedmeyer CE, Ferrario CM, Sowers JR. Mineralocorticoid receptor antagonism attenuates glomerular filtration barrier remodeling in the transgenic Ren2 rat. Am J Physiol Renal Physiol 2009; 296: F1013F1022.

17 Zhuo J, Ohishi M, Mendelsohn FA. Roles of AT1 and AT2 receptors in the hypertensive Ren-2 gene transgenic rat kidney. Hypertension 1999; 33: 347-353.

18 Watanabe D, Tanabe A, Naruse M, Morikawa S, Ezaki T, Takano K. Renoprotective effects of an angiotensin II receptor blocker in experimental model rats with hypertension and metabolic disorders. Hypertens Res 2009; 32: 807-815.

19 Valensi P, Assayag M, Busby M, Paries J, Lormeau B, Attali JR. Microalbuminuria in obese patients with or without hypertension. Int J Obes Relat Metab Disord 1996; 20: 574-579.

20 Tomaszewski M, Charchar FJ, Maric C, McClure J, Crawford L, Grzeszczak W, Sattar N, Zukowska-Szczechowska E, Dominiczak AF. Glomerular hyperfiltration: a new marker of metabolic risk. Kidney Int 2007; 71: 816-821. 